\title{
New insight into solvent engineering technology from evolution of intermediates via one-step spin-coating approach
}

\author{
Yingke Ren ${ }^{1}$, Bin Duan ${ }^{2}$, Yafeng $\mathrm{Xu}^{2}$, Yang Huang ${ }^{2}$, Zhaoqian $\mathrm{Li}^{2}$, Linhua $\mathrm{Hu}^{2}$, Tasawar Hayat ${ }^{3}$, \\ Hongxia Wang ${ }^{4}$, Jun $\mathrm{Zhu}^{2 *}$ and Songyuan $\mathrm{Dai}^{1,2,3^{*}}$
}

\begin{abstract}
The anti-polar solvent technique is an effective way to improve the film quality in a perovskite solar cell. In this work, we reveal the reason why chlorobenzene (CBZ) plays an important role in controlling the crystallization process. By investigating the formation of intermediate phases in the precursor solution, we observed that the $\mathrm{CH}_{3} \mathrm{NH}_{3} \mathrm{I}$ (MAI)- $\mathrm{PbI}_{2}$-dimethylformamide (DMF) or $\mathrm{MAI}-\mathrm{PbI}_{2}$-dimethylsulphoxide (DMSO) adducts have not yet formed until washed with non-polar solvent. The accelerated formation of intermediate phase yields high crystalline perovskite layers. Rapid solvent evaporation and retarded perovskite crystallization in one-step method are efficient to obtain high-quality perovskite films. Consequently, MAI-PbI ${ }_{2}$-DMSO intermediate shows neat rod-like structure with high crystallinity, which eventually transforms extremely dense and uniform perovskite films.
\end{abstract}

Keywords: perovskite solar cell, rapid-transformation, intermediate phases, anti-polar solvent technique

\section{INTRODUCTION}

During the past years, organic-inorganic hybrid perovskite cells have attracted much attention and revolutionized the prospects of photovoltaic technologies. The power conversion efficiency (PCE) of perovskite device has undergone a rapid development from $3.8 \%$ to $22.1 \%$ since the first attempt to introduce organic-inorganic hybrid perovskite materials as the light absorber [1-10]. Such rapid increase in performance can be ascribed to its excellent properties, such as, ambipolar charge transport, high carrier mobility, long carrier diffusion, and large absorption coefficient $[11,12]$. Previous studies have uncovered that full surface coverage and low surface roughness are crucial for governing the device photovoltaic performance $[5,6]$. Therefore, to prepare high quality perovskite thin films, various methods have been developed, e.g., vacuum evaporation [6], vapor-assisted deposition [13-15], solvent engineering $[16,17]$ and solution-based techniques [18-20]. Among these methods, solvent engineering technology has proven to be an efficient way for producing dense perovskite layers in a one-step deposition. This methodology can make use of non-polar solvent to regulate the perovskite crystallization process, and thus it has been widely used to produce uniform and dense perovskite films. For instance, Cheng and co-workers [17] reported that the conventional onestep spin-coating method followed by dropping chlorobenzene (CBZ) could lead to accelerated deposition of highquality perovskite film. In contrast, Seok and co-workers found that the $\mathrm{CH}_{3} \mathrm{NH}_{3} \mathrm{I}$ (MAI)- $\mathrm{PbI}_{2}$-dimethylsulphoxide (DMSO) intermediate phase retarded the reaction between MAI and $\mathrm{PbI}_{2}$ [16]. Although the solvent engineering technology is a very useful experimental methodology, there remain two major challenges. First, the effect mechanism of the non-polar solvent on the precursor adducts which further governs the perovskite formation has not been systematically studied yet. Second, the reason why

\footnotetext{
${ }^{1}$ Beijing Key Laboratory of Novel Thin-Film Solar Cells, North China Electric Power University, Beijing 102206, China

${ }^{2}$ Key Laboratory of Novel Thin-Film Solar Cells, Institute of Applied Technology, Hefei Institutes of Physical Science, Chinese Academy of Sciences, Hefei 230031, China

${ }^{3}$ NAAM Research Group, Department of Mathematics, Faculty of Science, King Abdulaziz University, Jeddah 21589, Saudi Arabia

${ }^{4}$ School of Chemistry, Physics and Mechanical Engineering Queensland University of Technology, Brisbane QLD 4001, Australia

*Corresponding authors (emails: sydai@ipp.ac.cn (Dai S); zhujzhu@gmail.com (Zhu J))
} 
the MAI- $\mathrm{PbI}_{2}-\mathrm{DMSO}$ adduct plays more important role in crystallization process than $\mathrm{MAI}-\mathrm{PbI}_{2}$-dimethylformamide (DMF) is unclear.

In this paper, we investigated the formation of MAI-PbI $-\mathrm{DMF}$ and $\mathrm{MAI}-\mathrm{PbI}_{2}$-DMSO intermediate phases by adding non-polar solvent (chlorobenzene) via a one-step coating method. The MAI-PbI ${ }_{2}-\mathrm{DMF}$ and MAI-PbI - -DMSO intermediate phases could not be found in the precursor solution until washed with CBZ. The results revealed that the non-polar solvent additive of CBZ improved the perovskite films in the following two aspects: (1) washed the excess solvent which is harmful for high-quality film formation; (2) promoted the formation of intermediate phases and thus retarded the crystallization of MAI and $\mathrm{PbI}_{2}$. Moreover, through studying the adducts formation condition with different solvents, surface topography, crystal structure and crystallization kinetics, we found that DMSO constituted MAI-PbI 2 -DMSO adduct showed neat rod-like structure and high X-ray diffraction peak intensity, leading to high-quality crystalline film. The perovskite solar cell annealed from $\mathrm{MAI}-\mathrm{PbI}_{2}-\mathrm{DMSO}$ adduct achieved a PCE up to $16.9 \%$.

\section{EXPERIMENTAL SECTION}

\section{Solar cell fabrications}

Fluorine-doped tin oxide (FTO) glasses (Pilkington, TEC15) were etched with $0.8 \mathrm{~mol} \mathrm{~L}^{-1} \mathrm{HCl}$ aqueous and $\mathrm{Zn}$ powder. The etched glasses were cleaned with detergent, distilled water and ethanol, and sonicated for $30 \mathrm{~min}$. By means of spray pyrolysis, the blocking $\mathrm{TiO}_{2}$ layers (bl- $\mathrm{TiO}_{2}$ ) were deposited on the as-prepared FTO which was followed by heating at $510^{\circ} \mathrm{C}$ for $30 \mathrm{~min}$.

The mesoporous $\mathrm{TiO}_{2}\left(\mathrm{mp}-\mathrm{TiO}_{2}\right)$ film was deposited on cooling bl- $\mathrm{TiO}_{2}$ by spin-coating of the $\mathrm{TiO}_{2}$ paste (Dyesol $30 \mathrm{NR}-\mathrm{T}$ ) following by calcining at $510^{\circ} \mathrm{C}$ for $20 \mathrm{~min}$.

$530 \mathrm{mg}$ of $\mathrm{PbI}_{2}$ and $183 \mathrm{mg}$ of $\mathrm{CH}_{3} \mathrm{NH}_{3} \mathrm{I}$ were dissolved in $1 \mathrm{~mL}$ of solvent containing different ratios of DMSO and DMF $(v / v)$, and then the solution was under magnetic stirring for $1 \mathrm{~h}$. The completely dissolved solution was spin-coated onto the $\mathrm{mp}-\mathrm{TiO}_{2}$ layer at $1100 \mathrm{rpm}$ for 10 $\mathrm{s}$ and $5000 \mathrm{rpm}$ for $30 \mathrm{~s}$. $0.75 \mathrm{~mL}$ of $\mathrm{CBZ}$ was quickly dripped onto the rotating substrate at 2 and $25 \mathrm{~s}$ after the onset of the second step (5000 rpm for $30 \mathrm{~s}$ ) for S-0 and S-100 solution, respectively. The surface would turn turbid in absence of CBZ washing. The perovskite film was heated at $100^{\circ} \mathrm{C}$ for $20 \mathrm{~min}$ in order to obtain a dense film. Then, spiro-OMeTAD solution $(25 \mu \mathrm{L})$, which consisted $73 \mathrm{mg}$ of spiro-OMeTAD, $28 \mu \mathrm{L}$ of 4 -tert-butyl pyridine and $17.5 \mu \mathrm{L}$ of lithium bis(trifluoromethanesulfonyl)imide (Li-TFSI) solution (520 mg of Li-TSFI in $1 \mathrm{~mL}$ of acetonitrile) in $1 \mathrm{~mL}$ of CBZ, was spin-coated on the perovskite film at $3000 \mathrm{rpm}$ for $20 \mathrm{~s}$. Finally, Au electrode with a thickness of $60 \mathrm{~nm}$ was deposited by using thermal evaporation under vacuum at a constant evaporation rate of $0.6 \mathrm{~nm} \mathrm{~s}^{-1}$.

\section{Characterization}

The morphology of the perovskite film was studied by a field emission scanning electron microscope (FESEM, FEI Sirion 200, Netherland). The crystal phase was obtained with X-ray diffraction (XRD, X'Pert Pro, Netherland) using $\mathrm{Cu}$ Ka beam $(\lambda=1.54 \AA$ ) $)$. The photocurrent density-voltage $(J-V)$ curves were measured under one sun illumination (AM 1.5G, $100 \mathrm{~mW} \mathrm{~cm}^{-2}$ ) with a solar simulator (94043A, USA) equipped with Keithley 2400 source meter. When measuring, a mask with $0.09 \mathrm{~cm}^{2}$ aperture area was used to avoid light scattering through the sides and define the effective area of the device. Fourier transform infrared spectroscopy (FTIR, Thermo Fisher IS50R, USA) was used to collect the spectral data for various solution and intermediates in the range of $4000-600 \mathrm{~cm}^{-1}$.

\section{RESULTS AND DISCUSSION}

In this work, to clarify the solvents containing DMF and DMSO with different ratios, we named S-0, S-15, S-30, S-70, S-85, and S-100 solution in the following discussions. These numbers represent the percentage of DMSO in the mixed solvent. The $\mathrm{P}-0$ and $\mathrm{P}-100$ powders were obtained by adding large amount of CBZ into the S-0 and S-100 solution and then drying in vacuum oven for $24 \mathrm{~h}$. Furthermore, the precursor (S-0 and S-100) was spin-coated onto a glass substrate. During spinning, CBZ $(0.75 \mathrm{~mL})$ was dripped at the center of the substrate and then the film was crystallized at $100^{\circ} \mathrm{C}$ for $20 \mathrm{~min}$. The corresponding perovskite films were named as F-0, F-100 films, respectively.

The pure DMF (S-0) solution precursor lead to the rod shaped structure of $\mathrm{MAPbI}_{3}$, which resulted from the different crystal growth rate between $\mathrm{MAI}$ and $\mathrm{PbI}_{2}$ [21]. In contrast, the rod-like structure of $\mathrm{MAPbI}_{3}$ disappeared if washed with non-polar solvent of CBZ during spinning the S-0 solution (Fig. 1a, b). Although washing with CBZ could improve the quality of $\mathrm{MAPbI}_{3}$ films, pinholes still could be observed, which agreed well with previous reports [21,22]. Similarly, as shown in Fig. 1c, d, when no CBZ was applied during pure DMSO (S-100) solution precursor spinning process, large gaps existed in perovskite films. The resulted perovskite film with large gaps might be caused by 

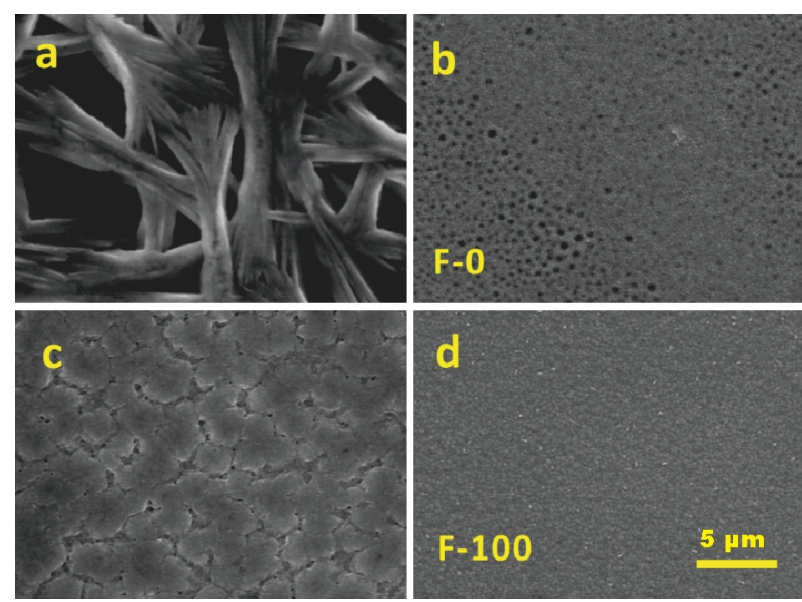

Figure 1 Planeview SEM topography for the perovskite films prepared by (a, b) S-0 solution and (c, d) S-100 solution. During the film spinning, the substrates (b) and (d) were treated with CBZ.

the large amount of DMSO in the precursor films. In comparison, the S-100 solution precursor spinning followed by CBZ drop-casting lead to smooth and homogeneous perovskite layers. These results indicate that washing with $\mathrm{CBZ}$ can control the evaporation rate of DMF or DMSO solvent. The excessive DMSO being washed off by CBZ produces more smooth and homogeneous $\mathrm{MAPbI}_{3}$ films since DMSO has stronger coordinate ability with $\mathrm{MAPbI}_{3}$ than DMF [23].

To further reveal the effect of $\mathrm{CBZ}$ on the perovskite crystallization process during the S-0 solution spinning, FTIR of DMF solvent, S-0 solution and P-0 powder were measured respectively. From Fig. 2a, b, we can see that the stretching vibrations of $\mathrm{C}=\mathrm{O}$ are located at $1661 \mathrm{~cm}^{-1}$ in DMF solvent and S-0 solution, but shifted to lower wavenumber of $1640 \mathrm{~cm}^{-1}$ in the $\mathrm{P}-0$ powder, which is originated from the weak $\mathrm{C}=\mathrm{O}$ bond strength interaction with
$\mathrm{PbI}_{2}$. Furthermore, $\mathrm{N}-\mathrm{H}$ stretching and bending reveal that the P-0 powder includes MAI molecules [16,24]. From these results, it can be seen that no adduct formation in S-0 solution in absence of $\mathrm{CBZ}$, while MAI-PbI ${ }_{2}-\mathrm{DMF}$ adduct resulted from $\mathrm{CBZ}$, which will retard the crystallization of MAI and $\mathrm{PbI}_{2}$ during the annealing. These results are in good agreement with the results observed by SEM (Fig. 1a, b).

Unstable MAI- $\mathrm{PbI}_{2}-\mathrm{DMF}$ intermediate has been recognized as the main reason for the pinhole on the perovskite film [21]. Therefore, evolution of the FTIR for DMSO solvent, S-100 solution and P-100 powder were further investigated. As shown in Fig. 3, the $\mathrm{S}=\mathrm{O}$ bond strength is located at $1042 \mathrm{~cm}^{-1}$ in DMSO solvent, but shifted to lower wavenumber of $1018 \mathrm{~cm}^{-1}$ for the S-100 solution and P-100 powder due to the interaction with the Lewis acid $\mathrm{Pb}^{2+}$ $[21,25]$. Moreover, the FTIR spectra of MAI-DMSO, $\mathrm{PbI}_{2}-$ DMSO and S-100 solution are provided in Fig. S1 for the functional group comparison. Although $\mathrm{N}-\mathrm{H}$ stretching and bending could not be found in FTIR spectra of MAIDMSO and S-100 Solution, it appears in P-100 powder which indicates the formation of $\mathrm{MAI}-\mathrm{PbI}_{2}$-DMSO adduct $[16,24]$. In other words, $\mathrm{PbI}_{2}$-DMSO adduct exists in S-100 solution and transforms into MAI-PbI ${ }_{2}$-DMSO adduct after CBZ addition, which will inevitably influence the quality of perovskite films. The $\mathrm{PbI}_{2}$-DMSO adduct in S-100 solution leads to large gaps on the perovskite film while the $\mathrm{MAI}-\mathrm{PbI}_{2}$-DMSO adduct results in smooth and homogeneous perovskite layer. FTIR spectra of other samples, such as, S-15, S-30, S-70 and S-85 are also provided in Fig. S2 to compare the functional group. The $\mathrm{PbI}_{2}$-DMSO intermediate adduct appears even at a low volume ratio of DMSO (S-15), and becomes more apparently with the DMSO ratio increasing, further confirming that DMSO has stronger coordination ability with $\mathrm{PbI}_{2}$ than DMF.
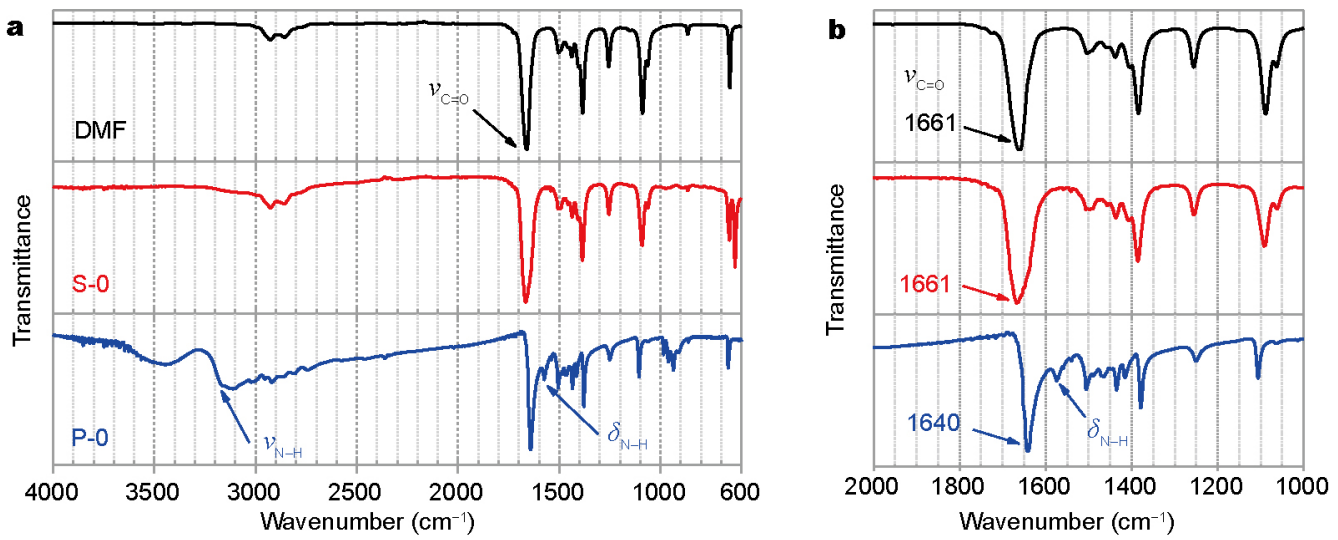

Figure 2 The FTIR of (a) DMF solvent, S-0 solution, P-0 powder and (b) expanded fingerprint region for the $\mathrm{C}=\mathrm{O}$ vibrations. 

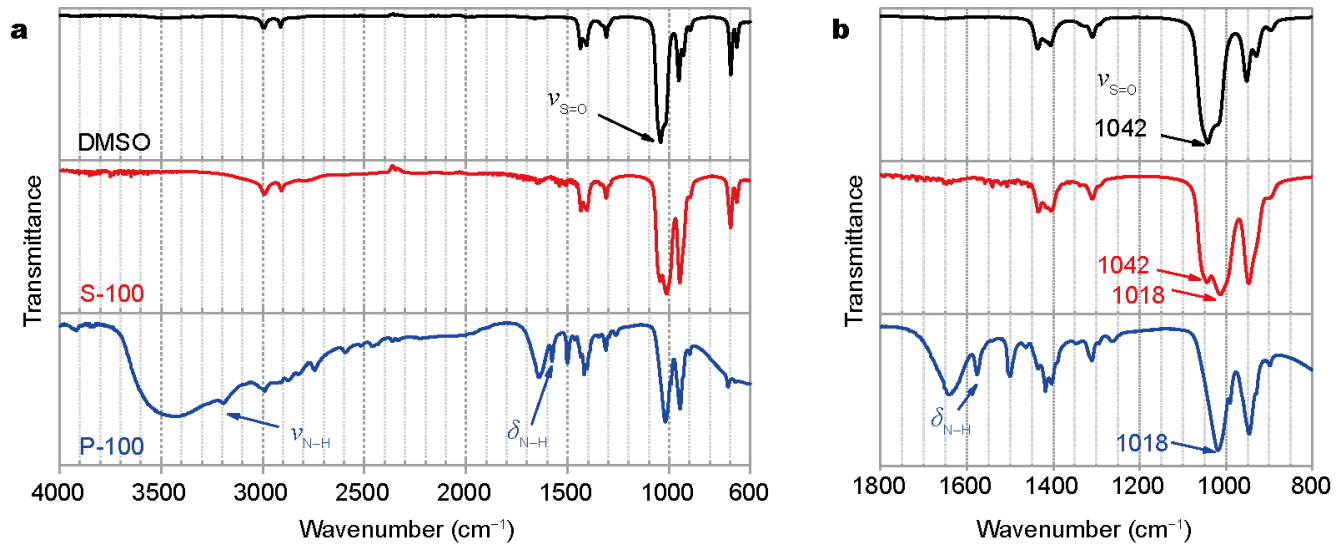

Figure 3 The FTIR of (a) DMSO solvent, S-100 solution, P-100 powder, and (b) expanded fingerprint region for the S=O vibrations.
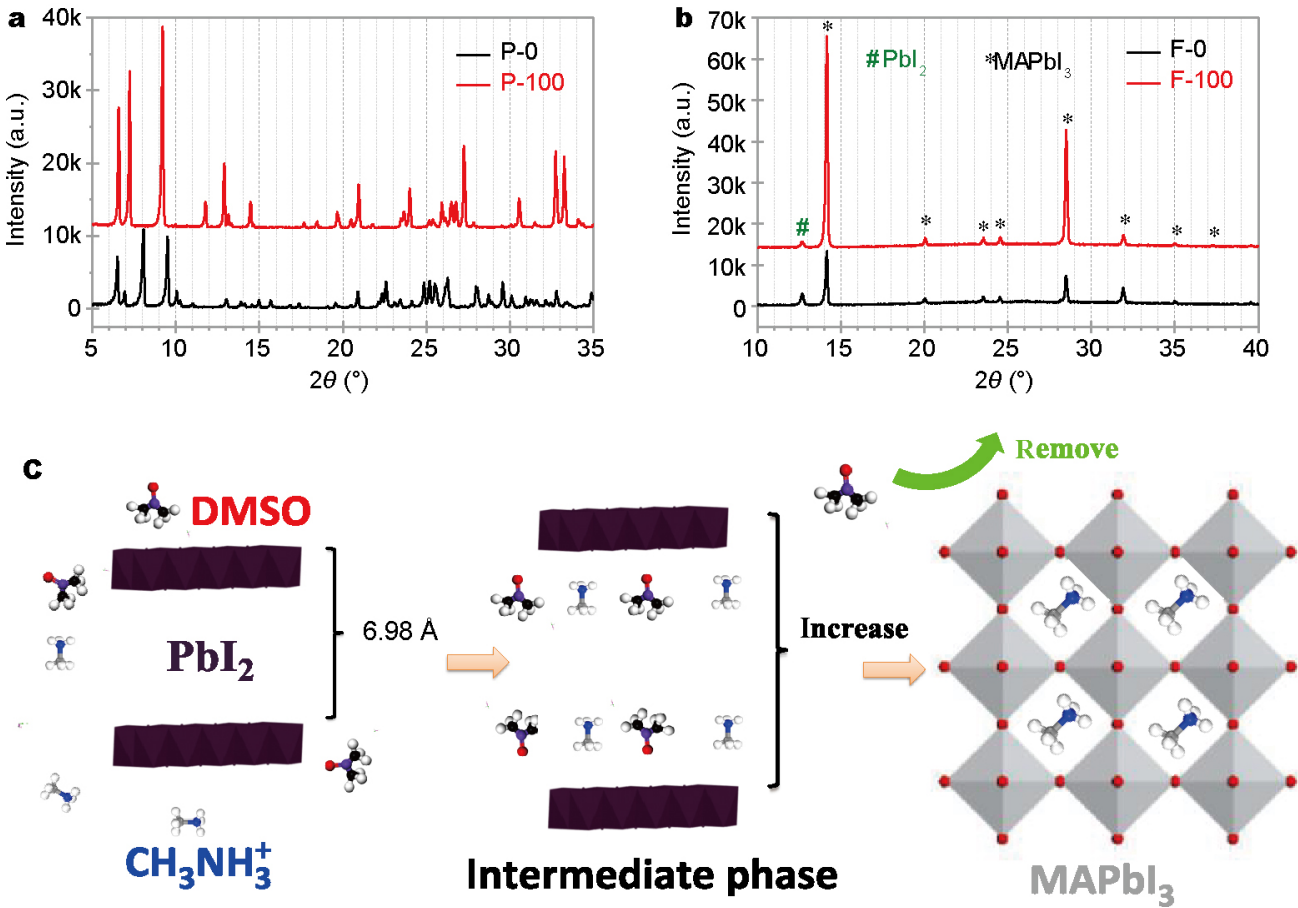

Figure 4 XRD patterns of (a) P-100 intermediate phases (b) and F-100 films. (c) Schematic models of the forming process of perovskite based on DMSO solvent.

On the basis of the above analysis, we can conclude that the MAI-PbI ${ }_{2}-\mathrm{DMF}$ and $\mathrm{MAI}-\mathrm{PbI}_{2}-\mathrm{DMSO}$ intermediate phases play an important role in the crystal growth and result in pinhole and uniform thin films, respectively. Thus, P-0 and P-100 powders were analyzed through XRD measurement and compared with $\mathrm{PbI}_{2}$ to understand the effect of underlying intermediate phase on the crystallization of perovskite films. The MAI, $\mathrm{PbI}_{2}, \mathrm{PbI}_{2}$ - $\mathrm{DMF}$ and $\mathrm{PbI}_{2}$-DMSO powers were characterized using $\mathrm{XRD}$, and the reflection pattern is presented in Fig. S3. A strong peak appearing at $9.44^{\circ}\left(\mathrm{PbI}_{2}-\mathrm{DMSO}\right)$ may be related to the presence of DMSO molecules in $\mathrm{PbI}_{2}$. Furthermore, the diffraction peaks at low angles $\left(6.55^{\circ}\right.$ and $7.23^{\circ}$, Fig. 4a) appear at P-100 intermediate phases. According to previous reports, the lower angle of intermediate phases indicates that MAI and DMSO molecules have intercalated into the $\mathrm{PbI}_{2}$ layered structure [18]. Based on the Bragg formulation, XRD peak of $\mathrm{PbI}_{2}$ (Fig. S3) at $12.66^{\circ}$ indicates that the interlayer spacing of the $\mathrm{PbI}_{2}(001)$ layered structure is approximately $6.98 \AA$, while in the MAI-PbI ${ }_{2}$-DMSO intermediate phases, the $\mathrm{PbI}_{2}$ lattice translation vector along the $c$ axis further increases. Af- 
ter heating at $100^{\circ} \mathrm{C}$ for $20 \mathrm{~min}$, the DMF and DMSO molecules inside the adducts tend to evaporate to produce perovskite. This mechanism could help us to understand the impact of intermediate phases on perovskite crystal growth. Namely, the crystallinity of intermediate phases is a crucial parameter on perovskite films [26,27]. The diffraction peak intensity of the MAI-PbI ${ }_{2}-\mathrm{DMSO}$ is much higher than that of MAI-PbI ${ }_{2}$-DMF intermediate phases. Consequently, XRD peak intensity of the $\mathrm{MAPbI}_{3}$ film induced by MAI- $\mathrm{PbI}_{2}-\mathrm{DMSO}$ is also much higher than that of $\mathrm{MAPbI}_{3}$ film induced by MAI-PbI ${ }_{2}$-DMF intermediate phases.

Analyses of these intermediate phases by SEM exhibit strikingly different morphologies. Fig. 5a shows fractured P-0 intermediate phase formed in the S-0 precursor solution, which easily loses DMF to transform $\mathrm{MAPbI}_{3}$. In contrast, S-100 solution produces P-100 fibers that grow preferentially along fixed directions (Fig. 5b). Considering the process of shrinking during annealing, only the dense intermediate phase could lead to the formation of perovskite films without pinholes and cracks (Fig. 1d). Thus, the perovskite films produced by MAI-PbI ${ }_{2}-\mathrm{DMSO}$ intermediate phase reveal full surface coverage.

Fig. 6a shows the typical $J-V$ curves for perovskite solar cells based on S-0 and S-100 solutions with and without drop-casting CBZ, respectively. Through comparing the photovoltaic parameters $\left(J_{\mathrm{sc}}, V_{\mathrm{oc}}, \mathrm{FF}\right.$ and PCE) followed by addition of CBZ, a remarkable increase in $J_{s c}$ and $V_{o c}$ was observed, which can be attributed to the realization of dense films. In addition, Since the S- 0 and S- 100 solutions could tune the grain morphology and might have a great effect on the device performance, here, perovskite solar cells were prepared by various solutions and the photovoltaic properties were investigated (Table 1). The perovskite device fabricated with S-0 solution washed with CBZ exhibits a PCE of $9.44 \%$ with the $V_{\text {oc }}$ of $0.93 \mathrm{~V}$, the $J_{\text {sc }}$ of $17.3 \mathrm{~mA} \mathrm{~cm}{ }^{-2}$, and $\mathrm{FF}$ of $58.7 \%$. In contrast, the S-100 solution washed with CBZ derived perovskite solar cell shows a significantly enhanced PCE of $16.9 \%$ with $V_{\mathrm{oc}}=$ $1.06 \mathrm{~V}, J_{\mathrm{sc}}=20.6 \mathrm{~mA} \mathrm{~cm}{ }^{-2}$, and $\mathrm{FF}=74.4 \%$. We endow this to the pinhole-free film morphology of the F-100 film. The smooth film would prevent direct contact between the Spiro-OMeTAD and $\mathrm{TiO}_{2}$. The corresponding incident photon-to-current efficiency (IPCE) for these cells further confirmed the $J_{\mathrm{sc}}$ results (Fig. 6b). In addition, Fig. S4 shows the typical $J-V$ curves for the perovskite solar cells base on S-0 and S-100 solutions measured by forward scan (FS) and reverse scan (RS), respectively. The $J-V$ curves of the forward and reverse scans (S-0 and S-100 solutions) were well coincident. These results reveal that the efficiency of the as-prepared perovskite solar cells is independent on the scanning direction. It is difficult to
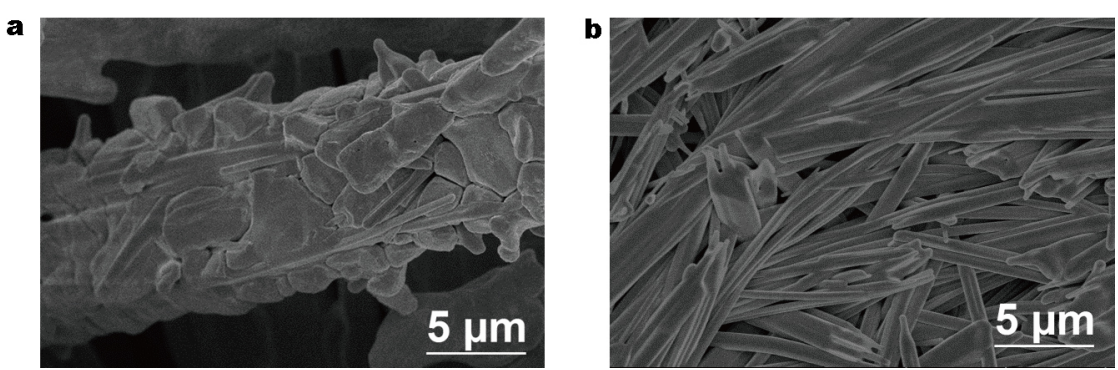

Figure 5 SEM images of (a) P-0 and (b) P-100 intermediate phases.
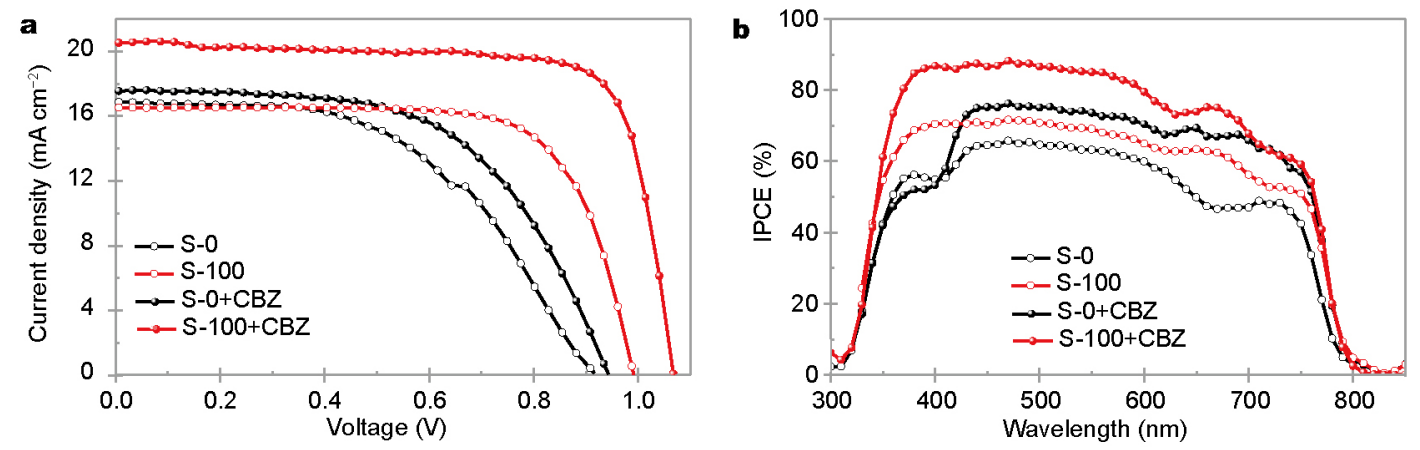

Figure $6 J-V$ curves of typical cells based on S-0 and S-100 solutions and (b) the corresponding IPCE spectra for the typical cells. 
Table 1 Photovoltaic properties of the perovskite solar cells prepared by various solutions

\begin{tabular}{ccccc}
\hline Solutions & $J_{\text {sc }}\left(\mathrm{mA} \mathrm{cm}^{-2}\right)$ & $V_{\text {oc }}(\mathrm{V})$ & FF $(\%)$ & PCE (\%) \\
\hline S-0 & 15.8 & 0.84 & 58.2 & 7.77 \\
S-0+CBZ & 17.3 & 0.93 & 58.7 & 9.44 \\
S-100 & 16.5 & 0.99 & 72.4 & 11.8 \\
S-100+CBZ & 20.6 & 1.06 & 77.4 & 16.9 \\
\hline
\end{tabular}

evaluate true device performance through the appearance of $J-V$ curves. Therefore, steady state output of best solar cell was further measured. As shown in Fig. S5, the typical solar cell exhibits stable steady state output during $100 \mathrm{~s}$ at a bias of $0.86 \mathrm{~V}$.

\section{CONCLUSION}

In summary, the effects of non-polar solvent (CBZ) on transformation of the intermediate phases were investigated. The high-quality perovskite films should be attributed to the rapid-transformed intermediates induced by non-polar solvent. The perovskite films grown using $\mathrm{CBZ}$ show dense surface coverage than the $\mathrm{CH}_{3} \mathrm{NH}_{3} \mathrm{PbI}_{3}$ layers without washing with CBZ. Through studying the surface topography and crystal structure of different adducts, we revealed that strong polar solvent of DMSO constituted MAI-PbI ${ }_{2}$-DMSO intermediate phase exhibited more neat structure, and the corresponding annealed films were uniform and dense. Consequently, the perovskite solar cell based on the MAI-PbI ${ }_{2}-\mathrm{DMSO}$ intermediate leads to a high PCE value up to $16.9 \%$. We hope this work will provide a new insight to understand the solvent engineering technology, and lead to perovskite solar cells with much higher PCE in the future.

Received 27 February 2017; accepted 29 March 2017; published online 17 April 2017

1 Kojima A, Teshima K, Shirai Y, et al. Organometal halide perovskites as visible-light sensitizers for photovoltaic cells. J Am Chem Soc, 2009, 131: 6050-6051

2 Kim HS, Lee CR, Im JH, et al. Lead iodide perovskite sensitized all-solid-state submicron thin film mesoscopic solar cell with efficiency exceeding 9\%. Sci Rep, 2012, 2: 591

3 Burschka J, Pellet N, Moon SJ, et al. Sequential deposition as a route to high-performance perovskite-sensitized solar cells. Nature, 2013, 499: 316-319

4 Liu M, Johnston MB, Snaith HJ. Efficient planar heterojunction perovskite solar cells by vapour deposition. Nature, 2013, 501: 395-398

5 Zhang W, Saliba M, Moore DT, et al. Ultrasmooth organic-inorganic perovskite thin-film formation and crystallization for efficient planar heterojunction solar cells. Nat Commun, 2015, 6: 6142

$6 \mathrm{Li} \mathrm{X}, \mathrm{Bi} \mathrm{D}, \mathrm{Yi} \mathrm{C}$, et al. A vacuum flash-assisted solution process for high-efficiency large-area perovskite solar cells. Science, 2016, 353:
58-62

7 Zuo C, Ding L. Modified PEDOT layer makes a $1.52 \mathrm{~V} V_{\text {oc }}$ for perovskite/PCBM solar cells. Adv Energ Mater, 2017, 7: 1601193

8 Zuo C, Bolink HJ, Han H, et al. Advances in perovskite solar cells. Adv Sci, 2016, 3: 1500324

9 Zuo C, Ding L. Bulk heterojunctions push the photoresponse of perovskite solar cells to $970 \mathrm{~nm}$. J Mater Chem A, 2015, 3: 9063-9066

$10 \mathrm{Li} \mathrm{ZQ}$, Chen WC, Guo FL, et al. Mesoporous $\mathrm{TiO}_{2}$ yolk-shell microspheres for dye-sensitized solar cells with a high efficiency exceeding 11\%. Sci Rep, 2015, 5: 14178

11 Xing G, Mathews N, Sun S, et al. Long-range balanced electronand hole-transport lengths in organic-inorganic $\mathrm{CH}_{3} \mathrm{NH}_{3} \mathrm{PbI}_{3}$. Science, 2013, 342: 344-347

12 Stranks SD, Eperon GE, Grancini G, et al. Electron-hole diffusion lengths exceeding 1 micrometer in an organometal trihalide perovskite absorber. Science, 2013, 342: 341-344

13 Huang F, Dkhissi Y, Huang W, et al. Gas-assisted preparation of lead iodide perovskite films consisting of a monolayer of single crystalline grains for high efficiency planar solar cells. Nano Energ, 2014, 10: 10-18

14 Zhang $\mathrm{M}$, Yu H, Yun JH, et al. Facile preparation of smooth perovskite films for efficient meso/planar hybrid structured perovskite solar cells. Chem Commun, 2015, 51: 10038-10041

15 Pascoe AR, Meyer S, Huang W, et al. Enhancing the optoelectronic performance of perovskite solar cells via a textured $\mathrm{CH}_{3} \mathrm{NH}_{3} \mathrm{PbI}_{3}$ morphology. Adv Funct Mater, 2016, 26: 1278-1285

16 Jeon NJ, Noh JH, Kim YC, et al. Solvent engineering for highperformance inorganic-organic hybrid perovskite solar cells. Nat Mater, 2014, 13: 897-903

17 Xiao M, Huang F, Huang W, et al. A fast deposition-crystallization procedure for highly efficient lead iodide perovskite thin-film solar cells. Angew Chem Int Ed, 2014, 53: 9898-9903

18 Shen D, Yu X, Cai X, et al. Understanding the solvent-assisted crystallization mechanism inherent in efficient organic-inorganic halide perovskite solar cells. J Mater Chem A, 2014, 2: 20454-20461

19 Hao F, Stoumpos CC, Liu Z, et al. Controllable perovskite crystallization at a gas-solid interface for hole conductor-free solar cells with steady power conversion efficiency over $10 \%$. J Am Chem Soc, 2014, 136: 16411-16419

20 Hao F, Stoumpos CC, Guo P, et al. Solvent-mediated crystallization of $\mathrm{CH}_{3} \mathrm{NH}_{3} \mathrm{SnI}_{3}$ films for heterojunction depleted perovskite solar cells. J Am Chem Soc, 2015, 137: 11445-11452

21 Ahn N, Son DY, Jang IH, et al. Highly reproducible perovskite solar cells with average efficiency of $18.3 \%$ and best efficiency of $19.7 \%$ fabricated via lewis base adduct of lead(II) iodide. J Am Chem Soc, 2015, 137: 8696-8699

22 Li G, Zhang T, Zhao Y. Hydrochloric acid accelerated formation of planar $\mathrm{CH}_{3} \mathrm{NH}_{3} \mathrm{PbI}_{3}$ perovskite with high humidity tolerance. J Mater Chem A, 2015, 3: 19674-19678

23 Wu Y, Islam A, Yang X, et al. Retarding the crystallization of $\mathrm{PbI}_{2}$ for highly reproducible planar-structured perovskite solar cells via sequential deposition. Energ Environ Sci, 2014, 7: 2934-2938

24 Yang WS, Noh JH, Jeon NJ, et al. High-performance photovoltaic perovskite layers fabricated through intramolecular exchange. Science, 2015, 348: 1234-1237

25 Lee JW, Kim HS, Park NG. Lewis acid-base adduct approach for high efficiency perovskite solar cells. Acc Chem Res, 2016, 49: 311-319

26 Li W, Fan J, Li J, et al. Controllable grain morphology of perovskite absorber film by molecular self-assembly toward efficient solar cell 
exceeding 17\%. J Am Chem Soc, 2015, 137: 10399-10405

27 efficiency perovskite solar cells via vapor induced intermediate phases. J Am Chem Soc, 2016, 138: 15710-15716

Acknowledgments This work was supported by the National Basic Research Program of China (2016YFA0202400 and 2015CB932200), the National Natural Science Foundation of China (21403247), the External Cooperation Program of BIC, Distinguished Youth Foundation of Anhui Province (1708085J09), Chinese Academy of Sciences (GJHZ1607), and STS project of Chinese Academy of Sciences (KFJ-SW-STS-152).
Author contributions Ren Y performed the main experiments and wrote this manuscript. Zhu J directly guided and conducted this research including the design, modifying and polishing work related to this manuscript. Dai S supervised the projects and carefully reviewed and modified this manuscript. Duan B, Xu Y, Huang Y, Li Z, Hu L, Wang H and Hayat $T$ provided help in the fabrication of devices and methods of characterization. All authors contributed to the general discussion about this work.

Conflict of interest The authors declare that they have no conflict of interest.

Supplementary information Supporting data are available in the online version of the paper.
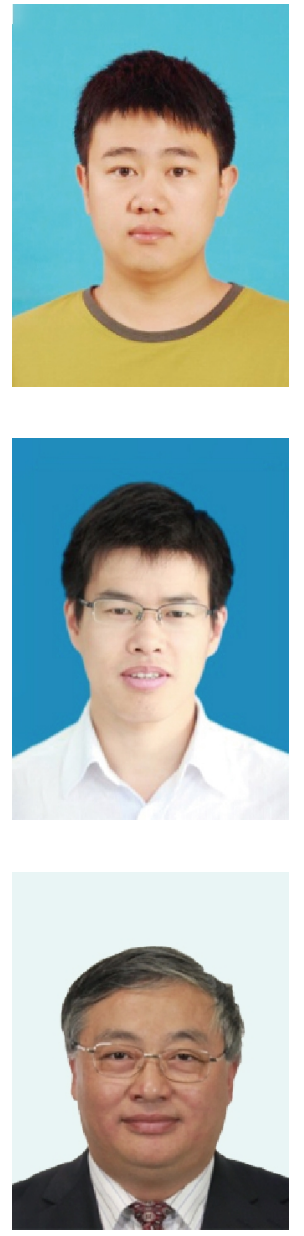

Yingke Ren obtained his BSc degree from Hebei Normal University in 2011. He is a PhD candidate of the North China Electric Power University under the supervision of Prof. Jun Zhu and Prof. Songyuan Dai. His research interests mainly focus on perovskite solar cells.
Jun Zhu received his PhD degree from the University of Science and Technology of China in 2005. He joined Hefei Institutes of Physical Science, Chinese Academy of Sciences and was promoted to full professor in 2015. Now his research interests focus on the new generation solar cells, including quantum dot solar cells and perovskite solar cells.
Songyuan Dai is a professor and Dean of the School of Renewable Energy, North China Electric Power University. He obtained his BSc degree in physics from Anhui Normal University in 1987, and MSc and PhD degrees in plasma physics from the Institute of Plasma Physics, Chinese Academy of Sciences in 1991 and 2001, respectively. His research interests mainly focus on the next-generation solar cells including dye-sensitized solar cells, quantum dot solar cells, perovskite solar cells, etc.

\section{一步旋涂法中反溶剂对中间相演变的新视角}

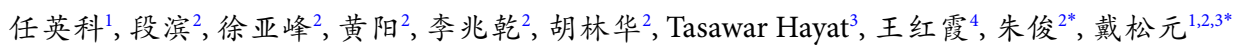

摘要 反溶剂技术可以有效改善钙钛矿薄膜的质量. 本文揭示了氯苯 (CBZ) 在结晶过程中起的重要作用. 通过研究前体溶液中配合物的结 构变化, 我们观察到, 在滴加反溶剂之前, MAI- $\mathrm{PbI}_{2}-\mathrm{DMF}$ 或MAI- $\mathrm{PbI}_{2}-\mathrm{DMSO}$ 尚未形成. 快速去除多余溶剂后形成中间相, 延缓钻钛矿结晶是 得到高质量钙钛矿薄膜的关键. 其中MAI-PbI 2 - $\mathrm{DMSO}$ 中间体表现出了高结晶的棒状结构, 因此最终转变成非常致密和均匀的钙钛矿薄膜. 